\title{
Del funcionalismo industrial al funcionalismo de servicios: ¡la nueva utopía de la metrópoli postindustrial del valle de México?**
}

\begin{abstract}
Old and new reflections regarding Mexico Valley's Metropolis are questioned, in order to discover new trends in urban development of the city. We assume the way in which we name the metropolis generates confusion. At the same time, there is an important transformation in the activities leading the economic development of the city. Services are taking the place that industrialization took for more than seventy years, currently adopting the leadership in the vision of the future of the postindustrial city. At the same time, natural and historical patrimonies are nowadays the main resources in order to revalue economic activities. Special mention has to be done to urban periphery, the place where growth and resources allow planning the sustainable city of the future.

Key words: Metropolis, megalopolis, deindustrialization, postindustrial city, natural patrimony, culturalpatrimony, urban periphery.
\end{abstract}

\section{Resumen}

El objetivo de este ensayo es responder algunas preguntas que han sido parte de viejas y nuevas reflexiones sobre la Metrópoli del Valle de México, y descubrir las nuevas tendencias que percibimos en su desarrollo. Partimos de suponer que hay confusión en la forma como se le nombra y que la visión de futuro sobre su transformación en el mediano y largo plazo pasa por un cambio importante de la función industrializadora que el modelo de sustitución de importaciones impuso a la ciudad, por otro de servicios y patrimonialista que le impone la visión postindustrial en que se ve inmersa en la actualidad. En esta transformación resalta la importancia de la periferia, dada la ubicación privilegiada que tiene en cuanto al patrimonio natural y cultural que le es propio, posibilitando así su contribución para alcanzar la sustentabilidad de la metrópoli.

Palabras clave: metrópoli, megalópolis, desindustrialización, ciudad postindustrial, patrimonio natural, patrimonio cultural, periferia urbana. 
"Las regiones de máxima convulsión y fragmentación son también las que parecen ser las que sobreviven mejor a los traumas de la devaluación en el largo plazo [...] Reindustrialización y reestructuración no pueden alcanzarse sin desindustrialización y devaluación primero.

(Harvey, 1989, p. 296)

\section{Introducción}

$\mathrm{L}$ a preocupación, siempre presente en el ámbito académico, por hacer un alto y evaluar los aportes de las investigaciones y los hallazgos que hemos tenido en nuestra tarea de rastrear los caminos por los que la Metrópoli del Valle de México ha incursionado en los últimos años, motivó a la Universidad Autónoma Metropolitana, a través de su Programa Universitario de Estudios Metropolitano, a realizar un seminario que permitiera "Repensar la metrópoli". La sesión introductoria tuvo como objetivo principal el análisis del contexto espacio-temporal en que se desenvuelve la transformación de la Metrópoli del Valle de México, poniendo especial énfasis en el papel y las acciones originadas en los ámbitos locales para analizar el impacto y la influencia que han tenido en la configuración y evolución de la misma ${ }^{1}$. La reflexión se orientó a partir de la pregunta siguiente: ¿qué papel han jugado los ámbitos locales en la configuración de la metrópoli?, independientemente de que en el desarrollo del trabajo aparecieron otras que reorientaron la reflexión y la discusión, entre las que se cuentan las siguientes: modelo de desarrollo territorial de la ciudad?, y de ser así, ¿cómo y hacia dónde?

Partiendo de la reflexión realizada y presentada como base para los debates del encuentro, el objeti-

* Departamento de Teoría y Análisis de la División de Ciencias y Artes para el Diseño de la UAM-Xochimilco. E-mail: bramirez@cueyatl.uam.mx

* Versión corregida y aumentada de la ponencia presentada en el Seminario Internacional: Repensar la Metrópoli, organizado por la Universidad Autónoma Metropolitana, México, 3-7 de octubre de 2005.

Se presentaron en esta sesión las contribuciones de Cruz (2005), Lerín (2005), Díaz y González (2005), Legorreta (2005) y Coulomb (2005), las que motivaron en gran parte la reflexión inicial con la que se pensó este trabajo. vo de este ensayo es responder a las preguntas anteriores, agregando otras que han sido parte de viejas y nuevas reflexiones sobre la Metrópoli del Valle de México. El objetivo fundamental que nos mueve es el de descubrir cuáles son las nuevas tendencias que percibimos en su desarrollo. Partimos de suponer que la visión de futuro sobre su transformación en el mediano y largo plazo pasa por un cambio importante de la función industrializadora que el modelo de sustitución de importaciones impuso a la ciudad, por uno de servicios y patrimonialista que le impone ahora la visión postindustrial en que aparentemente se ve inmersa como proceso. Asimismo, se resalta la importancia de las zonas periféricas como los entornos privilegiados para su desarrollo, enfatizando entonces la primacía de los agentes locales como los promotores y responsables de la transformación metropolitana.

Para efectos de la discusión, dividiré el ensayo en cuatro apartados. En el primero, me refiero a la forma como se ha transformado la caracterización de la ciudad en los últimos años, poniendo especial atención en las categorías que usamos para definirla y los debates que a partir de ahí se originan. En el segundo, trato de poner en evidencia los cambios de paradigmas que ahora se perciben para vislumbrar la posible dinámica futura de la metrópoli, y en el tercero reflexiono sobre la primacía que se da a la periferia como territorio de crecimiento y expansión y las nuevas funciones que se le adscriben. Por último, en una reflexión final, trataré de vincular las propuestas vertidas en los documentos presentados con el problema de la planeación y la utopía sobre la metrópoli, temas que son sin duda relevantes en relación a lo que queremos de nuestra sociedad, y por supuesto de nuestra ciudad.

\section{La definición metropolitana y megalopolitana}

Durante muchos años hemos intentado dar una explicación a la forma que adopta la ciudad, y para ello hemos usado diferentes categorías para analizarla y acercamientos teóricos para definirla. Por un lado ha existido la tendencia a explicar su crecimiento a partir de modelos de centralidad o geométricos que intentan dar orden a la forma como se organiza la población alrededor de centros tradicionales, históricos o de negocios y servicios 
(Burgess, 1988). Pero por otro, la teoría marxista intentó explicar el proceso urbano a partir de las formas urbanas que el capitalismo adoptó en su tendencia a la urbanización (Harvey, 2002). La diferencia existente entre forma-morfología y forma como proceso fue una de las tendencias que nos permitieron definir a la ciudad hasta la década de los ochenta del siglo pasado. En la primera se enfatiza su crecimiento, medido en términos de transformación demográfica e incremento en la superficie, y en la segunda, partiendo de los agentes y procesos de cambio, motivados por la producción capitalista desarrollada en las ciudades.

El funcionalismo adoptó esta visión de proceso y se encargó de asumir que la revolución industrial fue la causa fundamental de la urbanización contemporánea. En su proceso de crecimiento ampliado se adopta la categoría de metrópoli para caracterizarlo, hecho que se percibe tanto en Castells (1972) como en Unikel (1976) desde la década de los setenta, en un cambio de escala que requería el paso de la ciudad a su similar pero ampliada, llamada metrópoli.

En los últimos años hemos presenciado la adaptación de la categoría de megalópolis para denominar a las grandes aglomeraciones urbanas a las que se denomina también megaciudades (Ward, 1991), consideradas como "el rasgo característico del proceso de urbanización reciente”, la cuales se asocian a concentraciones mayores a seis u ocho millones de habitantes (Aguilar 2004, p. 5). Pero por otro lado, hay quienes las definen desde las actividades económicas que se han transformado en el curso de los procesos contemporáneos. Así, Sassen (2002) habla de las ciudades globales, definidas éstas a partir de las actividades terciarias que en ellas predominan, fundamentalmente las de servicios financieros y de informática y del proceso social de migración internacional que conforma ciudades multiculturales. La característica es que ambos, servicios y migración, están articulados a procesos y lugares globales.

Por último, hay quienes siguen definiendo la ciudad desde el punto de vista industrial-productivo a partir del paso de la producción fordista a la postfordista, que implica adecuarse a los nuevos cambios tecnológicos; pero también existe ahora la neofordista, que es la primera adecuada a la flexibili- dad que las condiciones actuales acreditan como fundamentales. En esta transformación la unidad territorial que nos sirve para explicarla es el de regiones urbano-globales, entendida en lo individual como "una masa central urbana rodeada por un hinterland que se extiende difusamente, salpicado por una red de centros urbanos discontinuos", y que se perciben, en términos geográficos, "como un mosaico o un archipiélago que se extiende al mundo entero" (Scott, 2002, p. 520).

Parecería entonces que en cada momento de la evolución social y del desarrollo urbano recurrimos como primera opción a denominar de manera diferente un crecimiento ampliado, que en todos los casos se acepta como dado y como cierto, en un cambio de escala que es complejo y que -en la opinión de algunos autores- no es preciso retomar, dada la concentración que persiste en los procesos de carácter metropolitano (Connolly y Cruz, 2004). Sin embargo, en mi opinión subsiste una falta de claridad en los conceptos que usamos para definir el proceso urbano, y en ocasiones lo usamos automáticamente sin llegar realmente a contextualizarlo y caracterizarlo, así como tampoco hemos discutido y definido en qué momento es conveniente y necesario, y para qué, el cambio de escala entre urbano, metropolitano y megalopolitano. Por ejemplo, hay autores que refiriéndose a la Metrópoli del Valle de México, caracterizan ya como megaciudad a Teotihuacan, la comparan con el crecimiento urbano de Nueva York y le adscriben implícitamente este carácter por el hecho de ser ciudades populosas (Díaz y González, 2005). Me pregunto si podemos usar la categoría de megaciudad para caracterizar a la urbe precolombina por el solo hecho de haber sido también populosa. Por otro lado, es preciso definir si populoso es lo mismo que muy poblado, y si eso hace que se inserte a la metrópoli en la categoría de megaciudad.

Por otro lado, sería interesante ver la diferencia que esta categoría tiene con la de megalópolis, considerada ya con problemas en un documento de Connolly (1999), en el que se argumentaba sobre la connotación de tamaño implícito en estas categorías, la inclusión o no de territorios considerados por unos autores y otros no, y la falta de bases teóricas que permitieran definirla. Contextualizar los procesos que son propios de la gran metrópoli del Valle de 
México es un problema complejo y difícil de definir por su dinámica espacio-temporal cambiante. Ya desde 1992, con la elaboración del Plan Regional del Valle de México, esta contextualización estaba marcada por dos escalas diferenciales del desarrollo de la ciudad: el metropolitano y el megalopolitano, la que es adoptada posteriormente por otros trabajos como el del Fideicomiso de Estudios Estratégicos sobre la Ciudad de México (2002), entre otros, y que -como veremos más adelante- marcan desde aquí una tendencia constante a definir, analizar e interpretar procesos metropolitanos a partir de un cambio de escala que no ha quedado claro si es justificado o no, o no hemos discutido para qué nos sirve y cuándo. Parecería que siguen más modas que se basan en la definición de nuevas categorías -como la de metápolis, por ejemplo- que definiciones de procesos reales de la urbanización contemporánea. En esta situación se encuentra también la caracterización de toda la metrópoli como global, cuando en realidad lo que se ha visto es que sólo algunos lugares de ella pueden ser considerados realmente como entornos globales integrados a los servicios internacionales o al comercio mundial, persistiendo entonces una metrópoli fragmentada que quizá tendría que denominarse de otra manera.

Otros criterios organizan su caracterización a partir de propuestas como las que se hacen en el Plan Nacional de Desarrollo Urbano y Ordenación del Territorio 2001-2006 (SEDESOL, 2001), que las integra en tres categorías: zonas metropolitanas, aglomeraciones urbanas y finalmente ciudades (Chávez y Guadarrama, 2004), a las que hay que agregar las de conurbación y otras. ¿Estamos hablando de categorías usadas para denominar al crecimiento o de las formas que éste adopta? Me parece que hay todo un trabajo pendiente que nos permita identificar claramente procesos sobre los que estamos hablando o saber si sólo queremos diferenciar formas que adopta la ciudad en diferentes momentos. Otra opción es pensar que, como investigadores, al mirar la ciudad observamos los procesos con miradas diversas, y por lo tanto destacamos tendencias que no coinciden, dados los objetivos que perseguimos al analizar la realidad, por lo que habría que llamarlas de diferente manera.

Parecería que en este cambio de nombres, el crecimiento urbano ampliado se toma ya como una situación irreversible, incontenible o bien hasta histórica y dada, asumiendo que la ciudad -ahora la metrópoli o la megalópolis- es un fenómeno que llegó para quedarse y que seguirá creciendo, aunque no tanto como se esperaba en los años setenta del siglo pasado, tal y como lo demuestran algunos trabajos sobre el tema (Partida y Anzaldo, 2004). Si el crecimiento es algo que está ya evidenciado, no hay nada más que decir sobre él, o bien está adoptando una nueva faceta que no estamos considerando como es el movimiento y la forma como incide directa o indirectamente en la dimensión territorial de la metrópoli. Algunos esfuerzos se han hecho para iniciar con este trabajo a nivel regional más que metropolitano (Ramírez, 2005a), pero requiere de una acercamiento diferente del tradicional, ya que por la falta de estadísticas que lo documenten, es cualitativo y no cuantitativo, y por lo tanto muy local, por lo que requiere de una investigación mayor que permita ubicarla en una dimensión no tan pequeña.

Se percibe también que proliferan los criterios utilizados para definir la expansión y la cantidad de municipios que se incluyen como metropolitanos ${ }^{2}$. Asimismo, jugamos con un cambio de escala para incluir su contexto en la megalopolitana, pero difícilmente tomamos acuerdos en relación a cómo identificarla y definirla (Connolly, Cruz, 2004), y mucho menos para poner en evidencia las definiciones locales y/o históricas que han incidido en este proceso. No es motivo de este ensayo el retomar estos criterios, pero sí señalar que, en ocasiones, en lugar de ayudar a las definiciones, más bien nos confunden. Un intento importante por tomar postura ante este problema se presenta en el documento de CONAPO y SEDESOL titulado "Delimitación de las zonas metropolitanas de México", publicado en 2004.

Si bien las asignaciones anteriores son usadas para definir el contexto del crecimiento urbano, en otros -como el de la sustentabilidad y el de la caracterización de los recursos naturales necesarios para su expansión, por ejemplo- resalta el uso de la categoría

Aquí hay una gran diferencia entre los 27 que considera el INEGI y los 58 que se incluyen en otros trabajos, además de otras propuestas donde el número de municipios metropolitanos varía dependiendo de los objetivos y alcances de cada trabajo y autor. 
de cuenca para denotar el ámbito en el cual se tienen que integrar y analizar los procesos vinculados con la metrópoli. Si bien refiere específicamente al ámbito hidráulico de conformación de una corriente fluvial, no queda del todo clara la pertinencia que tenga su uso para definir el entorno metropolitano desde el punto de vista económico y social. Sin embargo, al tratar temas específicos como el del agua, sin duda que su organización a partir de cuencas es importante para caracterizar la existencia del recurso y su posible utilización.

Por último, si bien el tema de la expansión ha sido y sigue siendo clave para la definición de la metrópoli, ésta se definía a partir de una centralidad única que marcábamos a partir del Centro Histórico de la ciudad. Sin embargo, en la actualidad hemos pasado a un paradigma policéntrico y difuso, que tiene varias formas de explicarse y de denominarse (Delgado, 1999; Scott, 2002; Aguilar, 2004). Connolly y Cruz (2004) han llamado la atención sobre la caracterización que se ha hecho en relación al contexto de crecimiento de la metrópoli, y demostrado en un texto reciente que el crecimiento mayor se da en los 30 municipios considerados ya como metropolitanos desde hace más de 20 años, dándole al proceso un carácter concentrado, a diferencia del difuso y centrífugo con el que lo caracterizan algunos estudios recientes (Aguilar, 2004). Asimismo, en relación al policentrismo con el que se identifica al crecimiento difuso y ampliado, se menciona que no es nuevo sino que tiene un carácter histórico, en la medida que se argumenta que ha sido una de las características adoptadas por la organización territorial del Valle de México desde la Colonia.

A lo anterior habría que agregar que ya en la década de los setenta, autores como Richardson (1971, p. 69), desde la teoría neoclásica, explican el origen de la generación de varios subcentros en la ciudad a partir de la crítica a la teoría concéntrica articulada a partir de los distritos centrales de negocios (lugares centrales), de la manera siguiente: "El análisis de los lugares centrales dentro de una metrópoli puede explicarlos, sobre todo si al extenderse la ciudad ha absorbido pequeños centros urbanos de fundación antigua. Pueden ser el reflejo de la gran demanda de espacio en ciertas actividades estratégicas (aeropuertos, universidades, etc.) que necesitan emplazamientos grandes pero compactos, demasia- do caros o imposibles de obtener cerca del centro; estas actividades actúan también como imán para otras actividades. Los establecimientos de servicio que abastecen un mercado que ya en sí sólo se encuentra en determinados puntos de la ciudad (por ejemplo, familias con rentas altas) también tenderán a localizarse lejos del centro. La escasez de emplazamientos céntricos y la posibilidad de que la accesibilidad sea menor pueden conducir en las ciudades muy grandes a la aparición de zonas secundarias de oficinas y centros comerciales suburbanos que formen nuevos núcleos. Hoy en día, cualquier ciudad grande está llamada a contener varios subcentros".

En suma, parece que vemos procesos nuevos en donde no los hay, y que no percibimos los que se siguen desarrollando, como el crecimiento y la concentración, pero sí evidenciamos nuevas categorías para definirlos. En otras ocasiones, usamos las propias de otros contextos y ciencias para definir el ámbito de crecimiento de la ciudad, como en el caso de cuenca. Me pregunto si no será que las teorías sobre las que basamos el estudio de la ciudad, o los modelos que usamos para explicar la forma que adoptan, están manifestando ahora su falta de correspondencia con lo que la realidad ha presentado históricamente, $\mathrm{y}$ no nos proporcionan las herramientas que necesitamos para entender y analizar la complejidad con la que se está articulando con los procesos contemporáneos. Quizá tengamos que utilizar menos nombres nuevos para denominar el crecimiento de la ciudad y poner más atención en los procesos reales y cambiantes y en la forma como los viejos y los nuevos se articulan, para completar una entramada red de posibilidades que no estamos definiendo.

\section{Cambio de paradigma en el modelo de desarrollo metropolitano: del funcionalismo industrial al terciario}

El funcionalismo metodológicamente nos legó una noción en la cual el crecimiento de la concentración urbana, ligado con el surgimiento de una economía fundamentalmente industrial, eran los planteamientos iniciales con los que empezábamos cualquier balance y diagnóstico sobre la metrópoli (Germani, 1988; Unikel, 1976). A esto se agregaba a veces la posibilidad de priorizar uno u otro (crecimiento o industria), resaltando sobre todo el sector dinámico que era este último (Garza, 1985). En la 
actualidad parecería que el contexto industrial del desarrollo urbano empieza a caer en desuso. Esto es evidente a pesar de que hay que reconocer que estudios sobre la configuración actual y los procesos territoriales vinculados con la industrialización han sido elementos de discusión en diferentes momentos y en diversos foros y seminarios. Sin embargo, en la evaluación del contexto local del desarrollo de la metrópoli, las transformaciones en el modelo de desarrollo se perciben a partir de dos supuestos: el cambio de la industrialización a la desindustrialización y la terciarización de la economía -y por lo tanto de la ciudad-, así como de la inclusión del patrimonio natural y cultural que le son propios, como elementos importantes para su reactivación económica. Desagreguemos un poco estos cambios.

Desde 1994, algunos autores llamaban ya la atención sobre la transformación económica de la metrópoli, donde la desindustrialización era una de sus manifestaciones más importantes (Hiernaux y Tomas, 1994). En 2002, el modelo de desarrollo de la ciudad fue caracterizado por el Fideicomiso de Estudios Estratégicos sobre la Ciudad en términos de desindustrialización, terciarización, informatización y contracción del mercado interno. Parecería que este cambio del secundario al terciario se ha expandido y ya se acepta como un hecho. Sus evidencias empíricas empiezan a ser discutidas en seminarios como el realizado recientemente en el Colegio de México, cuyo título orienta la importancia reciente de los impactos espaciales: "Organización espacial del sector servicios en México” 3 .

Este es un proceso que manifiesta algunas tendencias económicas que no son propias exclusivamente de la metrópoli del Valle de México, ya que la desindustrialización se empieza a observar en las metrópolis latinoamericanas desde los años ochenta del siglo pasado, siguiendo una trayectoria internacional que afecta a los principales centros industriales del mundo (Márquez, 2005). Se ve acompañada por un crecimiento importante del sector terciario, que en los países latinoamericanos adopta una forma de terciarización informalizada, por el gran crecimiento que ha adoptado el sector informal en nuestras latitudes con una manifestación territorial polariza-

3 Evento desarrollado el 21 y 22 de julio de 2005 . da entre el terciario moderno y el informal, y restringida al utilizar poca mano de obra y relativamente calificada.

Para algunos autores, este proceso constituye un "vaciamiento productivo" que es diferencial en las regiones del territorio mexicano, pero que sin duda, y coincidiendo con la tesis de Márquez (2005), ha afectado de manera directa y más intensa a la Zona Metropolitana del Valle de México, aunque lo ejemplifican también a nivel nacional (Quintana, Isaac y Nandayapa, 2006). Si bien son todavía pocos los trabajos que lo destacan, el de Márquez argumenta que también es un proceso diferencial en la Metrópoli del Valle de México, en virtud de que afecta con mayor intensidad al Distrito Federal que a los municipios conurbados del Estado de México (Márquez, 2005). En la opinión de la autora, el proceso se integra a partir de la medición de tres variables: personal ocupado, valor agregado y activos fijos, donde en el periodo 1980-1998 el Distrito Federal perdió $16,08 \%, 17,42 \%$ y 3,73 en cada uno de esos rubros, y los municipios conurbados perdieron un $5,17 \%$ y $6,77 \%$ en las dos primeras variables, ya que en la tercera se presentó un pequeño aumento de $0,31 \%$.

Para otros autores, las evidencias del proceso se manifiestan en los agudos cambios en el uso del suelo en los territorios estudiados, pero especialmente en lo municipios conurbados, como Ecatepec al norte de la metrópoli, a pesar de que no existe una cuantificación o cualificación más detallada del impacto que tiene al articularse con las actividades secundarias que, sin duda, persisten todavía en la ciudad. En relación con ello, el mismo autor menciona la falta de investigaciones que documenten el impacto y la transformación territorial de la producción postfordista que consideramos no es propia sólo del municipio de Ecatepec, sino que puede extenderse a toda la metrópoli del Valle de México y a otras de la República, ya que son pocas las investigaciones sobre el tema o bien representan casos fragmentados que no permiten reconstruir los cambios en una forma más integral.

En este proceso existen diferentes visiones que lo explicitan, ya que si bien para algunos autores la desindustrialización o el vaciamiento productivo es la forma característica que adoptan las metrópolis 
contemporáneas como parte de la crisis industrial que se presenta en la actualidad (Méndez, 1993 citado en Márquez, 2005), para otros se articula con el paso de la producción postfordista a una de carácter neofordista, esta última caracterizada por la recomposición de establecimientos fordistas hacia nuevas formas de producción más dinámicas y eficientes (Scott, 2002). Por otro lado, sería interesante aclarar si realmente para generar una reestructuración productiva es necesario primero desindustrializar para después recomponer, tal y como lo establece Harvey (1989) en su texto sobre la posmodernidad.

A pesar de que para algunos autores este cambio del secundario al terciario en las metrópolis es desafortunado, para otros como Coriat, la productividad de los servicios es un factor que permite no alarmarse, a pesar de ser inferior y más lento que el industrial (citado en Márquez, 2005). Esta argumentación se contrapone con la de los autores marxistas, quienes siguen sosteniendo que la generación de valor sólo se da en el secundario, y que este cambio incide directamente en la generación de la riqueza y por lo tanto en la productividad de la metrópoli. Aquí surgen nuevas preguntas: ¿cómo se está orientando la visión que estamos construyendo sobre la actividad fundamental de la metrópoli en los albores del siglo XXI?, y ¿cuál es el papel del sector terciario en esta visión?

Se vislumbra así un paso importante a nivel del paradigma contextual que rige el desarrollo metropolitano. Si antes se priorizaba la cualidad productiva industrial, ahora la primacía de los servicios y la informática son parte de la visión que se tiene de nuestro quehacer. Pero como no todos los ámbitos metropolitanos pueden insertarse en el contexto global de la informática y el comercio, el desarrollo económico local se percibe a partir del reconocimiento y explotación del patrimonio con el que cuenta cada parte de la metrópoli. Éste tiene dos maneras de implementarse: a partir del natural, constituido por los recursos y los lugares en donde la naturaleza todavía está presente y que permite hacer uso de ella, y el cultural, integrado por los bienes históricos de la ciudad.

De acuerdo con Díaz y González (2005, p. 11), y adscribiéndose a la reformulación de los documentos de la Carta de Venecia y de ICOMOS, el térmi- no patrimonio se refiere a "la riqueza y la diversidad del patrimonio cultural urbano que posee la Zona Metropolitana del Valle de México [que] representa un recurso muy valioso, del cual quisieran disponer otras ciudades, y que hasta ahora no ha sido ni suficientemente valorado, y menos aprovechado en beneficio de sus habitantes. Para ello, es indispensable consensuar entre los distintos poderes y niveles de gobierno, y con los organismos de la sociedad civil interesados, una re-conceptualización del patrimonio cultural, que supere la visión meramente conservacionista del mismo, concibiéndolo como un 'capital social', pero también como recurso no renovable, cuyo aprovechamiento puede ser la base de la sustentabilidad de su conservación" (subrayado de los autores).

Si bien se acepta y se hace un esfuerzo por superar las visiones meramente conservacionistas de lo cultural y de lo natural, ambas se ubican bajo el concepto de que producen valor, lo que les da la posibilidad de entrar en la reproducción del sistema económico de la ciudad, y así, son consideradas como parte de los bienes que inciden en el desarrollo económico y social de la metrópoli. Aunque se argumenta que hay "muchos elementos del patrimonio cultural urbano cuya preservación no obedece a la lógica de la ganancia” (Coulomb, 2005, p. 6), se dice también que es importante darles una vida útil (Díaz y González, 2006). Me pregunto: ¿cómo? Y no veo otra respuesta más que adscribir al patrimonio dentro de las actividades terciarias, entre las cuales se encuentra el turismo cultural y el ecológico.

Se argumenta que el patrimonio cultural más importante de la Metrópoli está en el centro histórico, aunque en la actualidad su manifestación territorial se dé como un corredor (Coulomb, 2005), pero que al abrirlo a la naturaleza y al patrimonio rural (cascos de ex haciendas, iglesias rurales, formas de producción rural como la de Xochimilco y Milpa Alta), en un cambio de escala importante, pierde su carácter central para ubicarse también en lo que se conoce como la periferia.

Si se considera exclusivamente como patrimonio histórico el Centro Histórico, a pesar de su enorme diversidad y gran diseminación dentro del territorio, el patrimonio cultural urbano tiene como soporte de apropiación colectiva una práctica socio- 
espacial fuertemente monocéntrica, que se pretende abrir para llegar a un policentrismo cultural que incluya también otros centros, y por supuesto, lo que consideramos como periferia. Esta necesidad de generar centros polifuncionales es parte de la consideración del patrimonio como elemento que estructure la ciudad, con un plan consensual que contemple una amplia gama de entornos que den nuevas centralidades a la ciudad. Es en esta policentralidad de los patrimonios que se descubre el carácter local de su ubicación, y por lo tanto, uno de los elementos más importantes en la reestructuración de la economía de la ciudad. Este supuesto tiene dos consideraciones: una donde es el propio habitante de las zonas con recursos quien podrá valorar su patrimonio, sea éste natural o cultural, para bien de la propia localidad, pero también otro donde se asume que el turista será también el propio habitante de la ciudad, con lo que será la población local quien valorice la utilidad del patrimonio en cuestión. Con ello, el agente fundamental de la transformación económica de la ciudad pasa a ser el habitante o el ciudadano, deslindando al Estado de proporcionar las condiciones necesarias para generarlo ${ }^{4}$.

En esta visión, ya no sólo el comercio y el crecimiento demográfico, sino también el uso útil del patrimonio, podrían contribuir en la construcción del "nuevo policentrismo", tanto de la ciudad como también de la metrópoli. Me pregunto, ¿este énfasis en las diferentes visiones del terciario cultural, impulsado tanto por el Fondo Monetario Internacional como también ahora por la UNESCO y el ICOMOS, quienes proporcionan buena parte de los recursos para acondicionar el patrimonio, no supone una especialización económica peligrosa para las ciudades, que implica aceptar que nuestra "vocación económica” ya no es la productiva, agrícola ni industrial, como se nos prometió con el modelo de sustitución de importaciones, desde que ahora sólo podemos reproducirnos a través de las actividades terciarias, específicamente las turísticas?

4 No entramos ahora a este debate, crucial en la vinculación entre Estado y habitante ciudadano para la transformación de la metrópoli. Lo dejamos acotado como uno importante para la generación de responsabilidades sobre el desarrollo.
Independientemente de la importancia que tenga el valorar el patrimonio, lo cual me parece muy válido, si el giro implica abandonar el resto de nuestras potencialidades, me parece que estamos ante una nueva división del trabajo internacional, donde nuestra tarea es dejar que produzcan los países del norte y aceptar que los sureños sólo podemos valorar nuestro patrimonio y cultura para beneficio local pero también global, pues seremos promotores del turismo a nivel internacional. Si esto es cierto, la geoestrategia de desarrollo local que estamos vislumbrando y aceptando deja desprotegida la base productiva de nuestra metrópoli, con lo cual el peligro de incrementar la dependencia y subordinación del exterior, que hasta ahora ha caracterizado el desarrollo de México, podría incrementarse. Si la modernización que nos propusimos en la primera mitad del siglo XX implicó aceptar que la "vocación" agrícola era limitada en aras de adoptar una industrial y urbana que también entró ya en crisis, no abandonemos ahora el poco o mucho potencial industrial que construimos y consolidamos en setenta años, en aras de asumir la moda patrimonialista como única forma de valorar la economía de la Metrópoli del Valle de México.

Pero también el patrimonio tiene una dimensión natural, que para autores como Lerín (2005) entra dentro de la asimilación de los recursos naturales y humanos, como parte de un discurso de sustentabilidad que los asimila al proceso de reproducción y expansión económica en la búsqueda de un equilibrio perdido y una posibilidad de sostener económicamente a la metrópoli. Los espacios locales, en su opinión, son territorios que deben aceptar un compromiso ante las exigencias de competitividad y calidad de vida que tendrá repercusiones en el sistema ecológico mundial. Me pregunto si esta diferenciación entre sustentable -que es también sostenible, es decir, que puede mantenerse por sí mismo- es posible en una Metrópoli como la del Valle de México, y si es lo mismo manejarla en la escala intra-urbana o en la regional de la periferia. El autor maneja posibilidades homogéneas en espacios no sólo diversos, sino con escalas de manejo territorial diferente, lo que amerita quizá tratarse de forma diferenciada. Se necesitaría incursionar en ejemplos locales que nos demuestren que esto es posible. 
Por último, en este cambio de paradigmas se redimensionan también categorías que usamos en sentidos varios, como son el paisaje cultural, que sustituye a la de región económica y la de patrimonio. Es preciso dar claridad a su uso para no perdernos en el camino de las indefiniciones o de las múltiples interpretaciones conceptuales, donde también aparece la necesidad de incluir la valorización del paisaje a través de su uso útil, que es el turismo ecológico.

En este redimensionamiento de los paradigmas con los que se explica el futuro de la ciudad, el crecimiento y futuro urbanos se explican todavía a partir de concebir un lugar central donde se sobre-enfatiza la función del abastecimiento de bienes y servicios (ahora turísticos y de renovación del patrimonio natural y cultural), simplificando el papel de las actividades terciarias para influir en la constitución de centros y de su hinterland. Ya Richardson (1971) argumentaba sobre estos planteamientos, que si bien son sugerentes, en muchos aspectos aparecen como rudimentarios y demasiado simplificados. Parecería entonces que empíricamente usamos y aplicamos elementos de la teoría del lugar central para proponer una alternativa policéntrica de desarrollo de la ciudad, que ya vimos que no funciona. Posiblemente no estemos concientes de este uso empírico e implícito de teorías que probaron ya su obsolescencia, pero que sugieren formas interesantes para una búsqueda válida sobre el desarrollo a futuro de la ciudad.

\section{La periferia metropolitana: expansión o zona de reproducción}

La periferia, reconocida por algunos autores como corona regional, periferia metropolitana o suburbio ${ }^{5}$, se ha convertido en el centro de atención del modelo sustentable y patrimonialista de la metrópoli, pues como se argumentó anteriormente, es ahí donde se encuentran tanto los espacios de posible crecimiento como los recursos que permitirán sostener el desarrollo en el presente y el futuro a partir de su aprovechamiento sustentable o patrimonial y su conservación. No sólo la denominación de este territorio, sino los procesos ahí desarrollados presentan también una gran cantidad de categorías con las que pretende-

\footnotetext{
5 Sobre las diferentes acepciones que tienen estos conceptos y los problemas que genera su uso indiscriminado, ver Ramírez (2005b).
}

mos caracterizarlos, entre los cuales se cuentan los de rururbanización, urbanización periférica u urbanización difusa, para nombrar las más usadas (Ramírez, 2005b). Clarificar el uso de estas categorías y darles un significado es también una tarea pendiente que ayudaría a definir no sólo procesos ubicados en esta zona, sino también la forma de nombrarlos apropiadamente.

En relación al crecimiento, en la opinión de Cruz (2005, p. 3), la urbanización popular contemporánea se expande a las "áreas rurales más cercanas a la metrópoli" a partir de tres formas: "la formación de numerosas colonias populares en el mercado de suelo informal, la construcción de conjuntos urbanos para los grupos sociales de bajos ingresos en terrenos con fuertes problemas para dotarse de los servicios urbanos básicos, y el crecimiento de los pueblos de la periferia metropolitana [...] Este intenso desarrollo del poblamiento popular involucra, por un lado, la existencia de una desigual relación con los recursos naturales existentes en la Zona Metropolitana del Valle de México, en la que la primacía de la lógica urbana subordina los procesos rurales y agrarios presentes en gran parte de la metrópoli. Por otra parte, el proceso que nos ocupa se ubica dentro de una de las dimensiones más problemáticas de la relación entre el ámbito urbano y rural, la urbanización de la propiedad ejidal y comunal".

Este vínculo entre propiedad de la tierra y urbanización ha sido un tema de gran importancia para reconocer los diferentes procesos con los que se produce el desarrollo urbano. Así, dice Cruz (2005), la urbanización periférica aparece cuando el crecimiento informal de la ciudad sobrepasó a la legislación y tuvo que regularizarse. Me pregunto si no es parte del crecimiento histórico de las ciudades, y la especificidad nuestra es que se dio a partir de la irregularidad. Ya Mumford (1964) hace mención de la importancia que tenían las áreas rurales y agrícolas que circundaban a la ciudad -suburbios, en su léxico-, como zonas que fueron amenazadas en su momento por el crecimiento urbano del entorno del crecimiento de Nueva York, donde ubica su estudio.

Sin embargo, a pesar de que hasta no hace mucho tiempo la urbanización periférica se había considerado parte de las formas de apropiación popular del territorio, en la actualidad hay una gran gama de 
posibilidades que las abren a urbanizaciones de clases medias y altas que salen ahora de la ciudad a los pueblos y lugares cercanos a éstas. En la actualidad ya también se incorporan a las zonas de urbanización popular el desarrollo de centros comerciales como el de Ecatepec, uno de los municipios más populares y populosos de la conurbación del Valle de México. Sin embargo, esta mezcla de lo popular con lo moderno es identificada por el autor como una forma de intervención de agentes globales en el territorio periférico popular. Creo que habría que desagregar y definir con mayor precisión la forma de identificar agentes locales y globales y la forma como inciden, deciden y marcan las transformaciones del territorio, quizá en una amalgama de decisiones que se toman en conjunto para realizarlos; y donde, aunque el modelo de centro comercial forma parte de una visión global de transformación territorial, requiere sin duda de decisiones y agentes locales para poder implementarse.

Este $\mathrm{u}$ otros temas nos permiten afirmar que la periferia metropolitana no se refiere a una unidad territorial homogénea, sino que se conforma como una donde las diferencias en la urbanización se agudizan por varias razones, entre las cuales se cuentan: la diferencia marcada en las condiciones físicogeográficas que la conforman (montaña en el occidente, ex lagos en el oriente); la mezcla tan variada de agentes que intervienen en su conformación y las diferentes gestiones que sobre los recursos y el territorio se presentan entre los municipios que la conforman. Esto nos permitiría preguntarnos: ¿hasta dónde hemos asumido un proceso de metropolización homogénea, que a nivel de lo local dista mucho de ser el que realmente hemos presenciado?, o ¿hasta dónde hemos estudiado la diferencia municipal de la urbanización sin integrar nuevamente el conjunto, de forma que nos permita reconstruir y percibir el proceso tan diversificado en el cual se ha desarrollado el crecimiento urbano de la metrópoli? Esto abre sin duda una agenda amplia de temas por reconstruir y trabajar en el futuro, y donde el papel local del sector inmobiliario y de otros agentes, no necesariamente populares pero también locales, tienen una gran importancia en el proceso de urbanización de la periferia metropolitana.

Pero además del crecimiento, la periferia es ahora el territorio preferido para localizar los elementos naturales necesarios para lograr la sustentabilidad de la metrópoli. La importancia que ésta adquiere aparece ante la inminente crisis que enfrenta un modelo de ciudad que se basó en el crecimiento irrestricto de las urbes, y que amenaza ahora con hacerlas no sólo insostenibles, sino irreproducibles y hasta vulnerables. El problema del agua es sin duda una de las dimensiones que demuestran más claramente el riesgo en que se encuentra la ciudad, de no tomar medidas rápidas para evitar la saturación del drenaje en la época de lluvias, entre otros muchos temas relacionados.

Particularizando sobre los recursos para la ciudad, el agua aparece como uno de los problemas sustantivos a remediar. Y me pregunto: ¿qué se está haciendo al respecto? :El implantar obras para retención y aprovechamiento del agua podrían ayudar a configurar una metrópoli diferente, y sobre todo sustentable, como se argumenta? Evidentemente, a nivel local esta solución tiene dos dimensiones: una es la del Estado, que tiene que ver con la propuesta de Legorreta (2005) en el sentido de resolver las fugas y la falta de captación en sistemas que almacenen las aguas torrenciales que nos inundan de forma de restaurar la naturaleza lacustre del valle. Pero me pregunto también si tendríamos que pensar y promover otra forma de uso del recurso que no estuviera basada en el mismo modelo de agua entubada al que hay que dar solución ante cualquier alternativa de crecimiento de la ciudad. Sin embargo, en las condiciones actuales, jse puede realmente pensar en volver a la ciudad de los lagos, como algunas propuestas sugieren? (Legorreta, 2005). Esto es algo que está en la mesa de la discusión y que tendríamos que considerar más en detalle, aunque -en opinión de algunos especialistas- sin duda que el problema tiene solución.

A esta agenda de temas pendientes sobre la periferia habría que agregar también la necesidad que hay de reconocer y analizar cuáles han sido las respuestas locales a la globalización, ya que en el contexto de los temas que a la fecha han sido trabajados parecería que son voces ausentes, no solamente en el reconocimiento de las estrategias económicas seguidas por la población para contender con el modelo neoliberal, sino también con relación a las formas que adopta la organización periférica y el papel que cumple en la urbanización metropolitana, a través 
de una mezcla de actividades que no siempre son reconocidas o aceptadas como importantes. La respuesta a la localización del aeropuerto alterno en Atenco es un ejemplo de movilizaciones de protección de los recursos naturales y económicos de los pobladores de la zona, que no podemos soslayar como visiones locales de desarrollo alterno de estas áreas. El rescate de la ruralidad y de algunas actividades con ella vinculados, que demandan algunos agentes locales de la periferia, y la importancia estratégica de mantener este espacio en producción, sería -entre otros- un tema también pendiente por desarrollar y considerar en trabajos y estrategias de la metrópoli a futuro.

\section{Reflexiones finales: organizar el futuro metropolitano y la planeación}

A pesar de las olas neoliberales que han permeado la concepción de las ciudades y el retiro del Estado que ello conlleva, se percibe un clamor general entre los académicos no sólo para hablar y proponer elementos de transformación de la metrópoli, sino también para reivindicar la importancia que tiene la planeación en el ordenamiento del futuro de la ciudad. Hay una insistencia por buscar su transformación en una forma mucho más organizada, en contraposición a la fragmentación y la diferenciación que se presentan en la actualidad; y a diferencia de lo que se podría pensar, hay una voluntad por buscar no sólo tendencias contemporáneas de transformación local, sino también propuestas de futuro a partir de reivindicar el papel de la planeación, como una actividad fundamental para poder dar ese orden al modelo de desarrollo.

Sin embargo, las propuestas que se presentan se caracterizan por contener una desarticulación de escalas que no permiten apreciar en conjunto cómo vislumbramos la evolución de la metrópoli, ya que en ocasiones priorizan la parte intra-urbana, pero en otras se refieren a connotaciones periféricas, o aun megalopolitanas, que impiden contender con una visión articulada de futuro ${ }^{6}$. Por un lado queda el Centro Histórico como un corredor cultural que revaloriza su imagen pero decrece en población, como parte fundamental de la visión de reconstitución del

6 Ver una propuesta metodológica para la articulación de estas escalas en Ramírez (2003). terciario en el centro de la ciudad. Por otro, persiste una desarticulación entre la ciudad de la producción y la del consumo, donde ahora la ciudad del patrimonio cultural y la necesidad de protegerlo "requiere de su incorporación a los procesos de ordenación territorial y de desarrollo urbano de la ciudad" (Coulomb, 2005, p. 2).

Pero al concebirse como "capital cultural", "el patrimonio cultural urbano representa un recurso no renovable que debe ser incorporado a la planeación estratégica de la Zona Metropolitana de la ciudad de México" (Coulomb, 2005, p. 2). Es evidente que este paso a la ordenación del patrimonio cultural es un factor fundamental a considerar en la planeación futura. Pero parecería que se encuentra desarticulada de la necesidad de contar también con una ordenación productiva y de servicios que asuman como igualmente importantes al consumo cultural para reproducir la ciudad y la producción industrial, y de otros servicios que en ella intervienen y se articulan. Queda pendiente la integración de estos sectores en una visión de futuro integrada donde se especifique las asignaciones y responsabilidades específicas tanto del sector privado como del público en la estrategia, así como el otorgamiento de prioridades dentro del conjunto. En este reajuste, en el nuevo papel del Estado en esta función de planeación parecería crucial su interacción con otros agentes que propicien también la transformación y el cambio. Este es sin duda un trabajo que nos queda todavía por realizar.

Asimismo, es necesario cuestionarnos si sólo lo viejo que se transforma en patrimonio y en valor permite y favorece que los territorios proporcionen identidad y cultura a la metrópoli, y si son sólo ellos los que pueden ser aprovechados y dinamizados por los agentes locales. A ellos se agregan sin duda los que funcionan como enclaves localizados, que parecería que no tienen cultura o la vinculan a la escala global, pero que sí tienen identidad y son importantes para la reproducción urbana. Me pregunto si acaso lo nuevo no genera también múltiples culturas locales, pero fragmentadas de las anteriores, y si acaso los jóvenes de clases medias y altas que viven y se recrean en estos lugares "sin cultura" no generan identidades con esos lugares. En ese sentido, y tomando la idea de De Mattos (1999) en relación a la necesidad de seguir considerando lo que existe y que 
sigue existiendo a pesar de lo nuevo, me pregunto: ¿cómo podemos vislumbrar desde lo local una ciudad no sólo fragmentada en dos, sino polivalente, dividida en múltiples visiones, que articula los diferentes elementos y escalas de la estructura económica y cultural para poder entenderla?

Esta complejidad, que pasa de la visión de la fragmentación dual a la multifragmentación, es algo que todavía no hemos percibido integralmente ni como un paradigma de acercamiento explicativo a los problemas de la metrópoli, y mucho menos como uno que permita proponer una planeación multifuncional, polifacética e integrante de diversas visiones y territorios. Por el contrario, parecería que ante la emergencia de procesos nuevos y viejos, pero más interrelacionados y a la vez fragmentados, para explicarlos seguimos recurriendo a viejos paradigmas, como el funcionalismo basado en una sola actividad o el lugar central, por ejemplo. Si a ello agregamos que seguimos considerando la planeación a partir de viejas prácticas que sólo vislumbran una estrategia para organizar el futuro de la ciudad, limitamos nuestra posibilidad de adentrarnos en el conocimiento y la organización de lo nuevo en las metrópolis a partir de un atraso conceptual y metodológico en nuestras prácticas.

Por otro lado, la importancia de la periferia abre sin duda la dimensión de la ciudad a otra escala, donde se perciben tres funciones principales: proporcionar suelo al crecimiento, conservar el patrimonio cultural y/o natural de la ciudad y proporcionar las condiciones que permitan la sustentabilidad o sostenibilidad de la metrópolis. Y cuando esta periferia deje de serlo y sea ciudad, me pregunto, ¿habría que recurrir a la escala siguiente (megalopolitana) para la obtención de lo necesario para la transformación de la ciudad? (Eibenzchutz, 1994). Nos olvidamos aquí de la necesidad de concebir lo local intraurbano y no sólo periférico como un elemento importante para vislumbrar la transformación futura de la ciudad, y una vez más introducir una propuesta más articulada que permita conjuntar estrategias internas y externas para resolver los problemas del futuro. Esto podría ayudar a concebir a la ciudad como un ente en continua expansión y que llegó para quedarse, pues al alcanzar una mayor dimensión, lo único que tenemos que asumir es un cambio de escala para percibirla y organizarla.
Por último, la crisis y visión catastrofista de la ciudad ha sido percibida en algunos documentos presentados, donde la insustentabilidad por deterioro económico, de civilidad y de calidad de vida (Fideicomiso de Estudios Estratégicos sobre la Ciudad, 2002) se agrega a problemas ecológicos por saturación del agua en drenaje causando inundaciones, lo que se caracteriza como vulnerabilidad hidrológica (Legorreta, 2005); a esto habría que agregar la crisis de los desechos sólidos que no ha tenido salida, profundizando problemas ya añejos que no sabemos en qué medida se han solucionado en el ámbito de lo local. Me parece que para resolverlos es necesario volver los ojos a miradas mucho más complejas que las que estamos fragmentando en la actualidad, integrando visiones y medidas que permitan evitar las catástrofes hidráulicas y económicas. Al mismo tiempo, es necesario buscar alternativas de revalorización del patrimonio y la producción, buscando integrar a los industriales activos y a otros actores como los campesinos, que conciben la posibilidad de su reproducción futura a partir de la ruralización de la ciudad y la reconstrucción de los cuerpos de agua (Legorreta, 2005). De esa manera, se podrá decir que sí trabajamos en la construcción de una ciudad de y para todos (Lerin, 2005).

\section{Referencias bibliográficas}

Aguilar, A.G. (2004). Procesos metropolitanos y grandes ciudades: dinámicas recientes en México y otros paises. México: UNAM, Instituto de GeografíaCRIM-CONACYT-Miguel Ángel Porrúa.

Burguess, E. (1988), "El crecimiento de la ciudad: introducción a un proyecto de investigación". Bassols, M., R. Donoso, A. Massolo, y A. Méndez, Antología de sociología urbana. México: UNAM, 118-129.

Castells, M. (1972). La cuestión urbana. Madrid: Siglo XXI.

Connolly, P. (1999). “¿Cuál megalópolis?” Delgado, J. y B. Ramírez (coords.), Territorio y cultura en la Ciudad de México. Tomo 1. México: Universidad Autónoma Metropolitana-Plaza Valdés, 37-46.

Connolly, P. y M.S. Cruz (2004). "Nuevos y viejos procesos en la periferia de la ciudad de México". Aguilar, A.G., Procesos metropolitanos y grandes ciudades: dinámicas recientes en México y otros 
países. México: UNAM, Instituto de GeografíaCRIM-CONACYT-Miguel Ángel Porrúa.

Cruz, M.S. (2005). ”Propiedad de la tierra y gestión del suelo urbano en la periferia metropolitana”. Contribución a la Mesa 1 del Seminario Repensar la Metrópolis, 3-7 de octubre. México: Universidad Autónoma Metropolitana.

Coulomb, R. (2005). "Patrimonio cultural urbano y desarrollo metropolitano". Contribución a la Mesa 1 del Seminario Repensar la Metrópolis, 37 de octubre. México: Universidad Autónoma Metropolitana.

Chávez, A.M. y J. Guadarrama (2004). “La región de México en transición: tendencias económicas y migratorias a finales del milenio". Aguilar, A.G., Procesos metropolitanos y grandes ciudades: dinámicas recientes en México y otros países. México: UNAM, Instituto de Geografía-CRIMCONACYT-Miguel Ángel Porrúa.

De Mattos, C. (1999). "Santiago de Chile, globalización y expansión metropolitana: lo que existía y sigue existiendo". EURE Revista Latinoamericana de Estudios Urbano Regionales, 25, 76 : 29-56.

Delgado, J. et al. (1999). "Estructura metropolitana y transporte". Eibenschutz, R. (coord.), Bases para la planeación del desarrollo urbano en la Ciudad de México. Tomo II. México: UAM-Miguel Ángel Porrúa.

Díaz, S. y A. González (2005). “De una expansión indiscriminada a una planeación que incluya y respete el patrimonio cultural y natural". Contribución a la Mesa 1 del Seminario Repensar la Metrópolis, 3-7 de octubre. México: Universidad Autónoma Metropolitana.

Eibenzchutz, R. (1994). “ ¿Ha cambiado la tendencia? Opciones para el desarrollo de la Ciudad de México”. Hiernaux, D. y F. Tomas (comps.), Cambios económicos y periferia de las grandes ciudades: el caso de la Ciudad de México. México: IFAL-Universidad Autónoma Metropolitana, 127-145.

Fideicomiso de Estudios Estratégicos sobre la Ciudad de México (2002). La Ciudad de México hoy. México: Gobierno del Distrito Federal, Oficialía Mayor.

Garza, G. (1985), El proceso de industrialización en la ciudad de México. México: El Colegio de México.
Germani, G. (1988), "La ciudad como mecanismo integrador”. Bassols, M., R. Donoso, A. Massolo y A. Méndez, Antología de sociología urbana. México: UNAM, 267- 286.

Harvey, D. (1989). The condition of posmodernity. London: Blackwell. (2002). "The urban process under capitalism: A framework for analysis”. Bridge, G. yS. Watson, The Blackwell city reader. London: Blackwell, 116-124.

Hiernaux, D. y F. Tomas (comps.) (1994). Cambios económicos y periferia de las grandes ciudades: el caso de la Ciudad de México. México: IFAL-Universidad Autónoma Metropolitana.

Legorreta, J. (2005). "El agua y la ciudad de México. De Tenochtitlán a la megalópolis del siglo”. Contribución a la Mesa 1 del Seminario Repensar la Metrópolis, 3-7 de octubre. México: Universidad Autónoma Metropolitana.

Lerín, M. (2005). "Sustentabilidad o sostenibilidad; arquitectura y metrópoli”. Contribución a la Mesa 1 del Seminario Repensar la Metrópolis, 37 de octubre. México: Universidad Autónoma Metropolitana.

Márquez, L. (2005). La desindustrialización del área metropolitana de la Ciudad de México, 19802004. Tesis presentada para obtener el grado de Maestra en Estudios Regionales. México: Instituto de Investigaciones Dr. José María Luis Mora.

Méndez, R. (1993). Procesos de reestructuración industrial en las aglomeraciones metropolitanas españolas. Madrid: Ministerio de Obras Públicas yTransportes.

Mumford, L. (1964). The highway and the city. Chicago: Mentor Book.

Quintana, L., J. Issac y C. Nandayapa (2006). "Encadenamientos productivos, industria y vaciamiento productivo regional en México". Ponencia presentada en el Seminario sobre Agenda del Desarrollo Nacional, 24 de marzo. México: Facultad de Economía, UNAM.

Partida, V.y C. Anzaldo (2004). "Escenarios demográficos y urbanos de la Zona Metropolitana del Valle de México". Aguilar, A.G., Procesos metropolitanos y grandes ciudades: dinámicas recientes en México y otros paises. México: UNAM, Instituto de Geografía-CRIM-CONACYT-Miguel Ángel Porrúa, 189-218. 
Blanca Ramírez

Ramírez, B. (2003). "Procesos territoriales, escalas y utopía”. Ciudades, 60:9-13.

(2005a). "La otra megalópolis: movilidad como cultura laboral". Anuario del Posgrado en Ciencias y Artes para el Diseño. México: UAMXochimilco (en prensa). (2005b), "Miradas y posturas frente a la ciudad y el campo". Ávila, H., Lo urbano-rural, ¿nuevas expresiones territoriales? México: Centro Interdisciplinario de Estudios Regionales, UNAM, 61-85.

Richardson, H. (1971), Economía del urbanismo. Madrid: Alianza.

Sassen, S. (2002). "From globalization and its discontents". Bridge, G y S. Watson (eds.), The Blackwell city reader. London: Blackwell, 160-170.
Scott, A. (2002), "Regiones urbano-globales. Dilemas de planeación y política en el mundo neoliberal”. Basave, J., A. Dabat, C. Morera, M.A. Rivera y F. Rodríguez (coords.), Globalizacióny alternativas incluyentes para el siglo XXI. México: UNAM-UAM- Miguel Ángel Porrúa, 513-566.

Unikel, L. (1976). El desarrollo urbano en México: diagnóstico e implicaciones futuras. México: El Colegio de México.

Ward, P. (1991). México megaciudad: desarrollo y politica, 1970-2002. México: Miguel Ángel Porrúa. 